\title{
Cosmetic surgeries on genus one knots
}

\author{
JIAJUN WANG
}

\begin{abstract}
In this paper, we prove that there are no truly cosmetic surgeries on genus one classical knots. If the two surgery slopes have the same sign, we give the only possibilities of reflectively cosmetic surgeries. The result is an application of Heegaard Floer theory and number theory.
\end{abstract}

57M27, 57R58; 53D40

\section{Introduction}

Let $Y$ be a closed oriented three-manifold and $K$ be a framed knot in $Y$. For a rational number $r$, let $Y_{r}(K)$ be the resulting manifold obtained by Dehn surgery along $K$ with slope $r$ with respect to the given framing. For a knot $K$ in $S^{3}$, we will always use the Seifert framing. Two surgeries along $K$ with distinct slopes $r$ and $s$ are called cosmetic if $Y_{r}(K)$ and $Y_{s}(K)$ are homeomorphic. The two surgeries are truly cosmetic if the homeomorphism is orientation preserving and reflectively cosmetic if the homeomorphism is orientation reversing. Cosmetic surgeries $Y_{r}(K)$ and $Y_{S}(K)$ are mundane if there is a homeomorphism of the knot exterior taking $r$ to $s$ and exotic otherwise.

Surprisingly, (reflectively) cosmetic surgeries are not rare at all. For an amphichiral knot $K$ in $S^{3}$, we always have $S_{r}^{3}(K) \cong-S_{-r}^{3}(K)$. Interesting examples are given by Mathieu [18]. He constructed a series of cosmetic surgeries on the trefoil $T$, namely, for any nonnegative integer $k$, we have

$$
S_{(18 k+9) /(3 k+1)}^{3}(T) \cong-S_{(18 k+9) /(3 k+2)}^{3}(T) .
$$

The resulting manifold $M_{k}$ is the Seifert fibred space which has Seifert invariant $S(0 ; k-3 / 2 ;(2,1),(3,1),(3,2))$ in the notation of Jankins and Neumann [14]. By results in Seifert fibred spaces, $M_{k}$ admits no orientation-reversing homeomorphisms. So they are not truly cosmetic. As pointed out in [3], the existence of Seifert fibred cosmetic surgeries highly depends on the existence of an exceptional fibre of index 2, which could be recovered by Rolfsen twist after orientation reversal.

An even more striking example is given by Bleiler, Hodgson and Weeks [3]. They give an oriented 1-cusped hyperbolic 3-manifold $X$ with a pair of slopes $r_{1}$ and $r_{2}$ such 
that the two surgered manifolds $X\left(r_{1}\right)$ and $X\left(r_{2}\right)$ are oppositely oriented copies of the lens space $L(49,18)$ (and there is no homeomorphism $h$ of $X$ such that $h\left(r_{1}\right)=r_{2}$ ). However, it is still unknown whether there are hyperbolic manifolds that could be obtained via exotic (truly or reflectively) cosmetic surgeries. A result in [3] indicates that such examples would be unexpected.

There are truly cosmetic surgery on the unknot: $S_{p / q}^{3}(U) \cong S_{p / p+q}^{3}(U)$ (mundane) and $S_{p / q_{1}}^{3}(U) \cong S_{p / q_{2}}^{3}(U)$ (exotic) where $q_{1} q_{1} \equiv 1(\bmod p)$. However, so far, there are no known truly cosmetic surgeries on a nontrivial knot. In fact, recall the following conjecture, Problem 1.81(A) in Kirby's problem list:

Conjecture 1.1 (Cosmetic Surgery Conjecture [1]) There are no truly cosmetic surgeries on a knot $K$ in a closed three-manifold $Y$ provided that the knot complement is not homeomorphic to the solid torus. In particular, for any classical nontrivial knot $K$ in $S^{3}, S_{r}^{3}(K) \cong S_{S}^{3}(K)$ if and only if $r=s$.

One approach to this conjecture is to get restrictions on manifolds that could arise as the resulting manifold of cosmetic surgeries. For example, the three-sphere (Gordon and Luecke [12]) and the manifold $S^{2} \times S^{1}$ (Gabai [10]) could not be obtained via cosmetic surgeries (truly or reflectively). In [29], Rong classified all Seifert fibered spaces that could be obtained by cosmetic surgeries on a knot whose complement is Seifert fibered and boundary incompressible. One remarkable consequence of Rong's classification is that if a knot $K$ with Seifert fibered complement admits a pair of cosmetic surgeries yielding a Seifert fibered space, then it admits infinitely many such cosmetic surgeries. All these cosmetic surgeries are reflectively cosmetic.

Another approach is to get restrictions on knots in manifolds that admit cosmetic surgeries. In [17], Lackenby showed that for two surgered manifolds $Y_{p / q}\left(K_{1}\right)$ and $Y_{p^{\prime} / q^{\prime}}^{\prime}\left(K_{2}\right)$, if $Y$ and $Y^{\prime}$ are distinct, or $K_{1}$ and $K_{2}$ are distinct, or $p / q \neq p^{\prime} / q^{\prime}$, then we have $Y_{p / q}\left(K_{1}\right) \not Y_{p^{\prime} / q^{\prime}}^{\prime}\left(K_{2}\right)$ provided that $\left|q^{\prime}\right|$ is sufficiently large. This implies that truly cosmetic surgeries are really rare.

Heegaard Floer theory was introduced by Ozsváth and Szabó in [27] and other sequels; see [26] for an overview. Heegaard Floer theory assigns invariants to closed threemanifolds (called Heegaard Floer homologies) and four-manifolds. And there are also numerous other invariants, such as knot Floer homologies and Ozsváth-Szabó contact invariants. Heegaard Floer theory (especially the rational surgery formula [23]) gives strong restrictions on (classical) knots that admit cosmetic surgeries. Roughly speaking, an $L$-space is a closed 3-manifold having the same Heegaard Floer homology as some lens space. In particular, we have the following theorem. 
Theorem 1.2 (Ozsváth-Szabó [23]) Let $K \subset S^{3}$ be a knot. If $r, s$ are distinct rational numbers and $S_{r}^{3}(K) \cong \pm S_{s}^{3}(K)$, then either $S_{r}^{3}(K)$ is an $L$-space or $r$ and $s$ have opposite signs. If $K$ is of Seifert genus one, and $S_{r}^{3}(K) \cong S_{s}^{3}(K)$ with $r \neq s$, then $S_{r}^{3}(K)$ is an $L$-space and $K$ has the same knot Floer homology (and hence the same Alexander polynomial) as the trefoil knot $T$.

In this paper, based on the above theorem, we prove Conjecture 1.1 for genus one classical knots. Specifically, our main result is the following theorem.

Theorem 1.3 For two Dehn surgeries with slopes $p / q$ and $p / q^{\prime}$ on a Seifert genus one knot $K$ in $S^{3}$, let $S_{p / q}^{3}(K)$ and $S_{p / q^{\prime}}^{3}$ be the resulting manifolds. Then

(1) $S_{p / q}^{3}(K) \cong S_{p / q^{\prime}}^{3}(K)$ if and only if $q=q^{\prime}$.

(2) If $S_{p / q}^{3}(K) \cong-S_{p / q^{\prime}}^{3}(K)$ and the slopes $p / q$ and $p / q^{\prime}$ have the same sign, then either $K$ has the same knot Floer homology as the right-handed trefoil knot and $p=18 k+9,\left\{q, q^{\prime}\right\}=\{3 k+1,3 k+2\}$ for some nonnegative integer $k$, or $K$ has the same knot Floer homology as the left-handed trefoil knot and $p=18 k+9,\left\{q, q^{\prime}\right\}=\{-(3 k+1),-(3 k+2)\}$ for some nonnegative integer $k$.

Our theorem guarantees that Mathieu's examples are the only cosmetic surgeries on the right-handed trefoil. In fact in their survey paper [26], Ozsváth and Szabó implicitly conjectured that knot Floer homologies detect fibered of knots in $S^{3}$, namely, a knot $K$ in $S^{3}$ is fibred if and only its knot Floer homology in the topmost nontrivial filtration is isomorphic in $\mathbb{Z}$. Several classes of knots have been verified for this conjecture: pretzel knots in Ozsváth and Szabó [28], some cable knots in Hedden [13], Whitehead double knots in Eftekhary [6], closed 3-braids in Ni [20], etc. See Ni [22] for theoretical evidence. So by Theorem 1.2, we expect that the reflectively cosmetic surgeries on the trefoil knot are the only cosmetic surgeries on genus one knots if the slopes have the same sign. (Of course on amphichiral knots, such as the figure eight knot, there are many reflectively cosmetic surgeries with slopes in opposite signs by letting $r=-s$.)

The ingredients in our proof of Theorem 1.3 are Heegaard Floer theory, Reidemeister torsion, the Casson-Walker invariant and number theory on cyclotomic numbers. Truly cosmetic surgeries of slopes with opposite signs on genus one knots are ruled out by Theorem 1.2. For cosmetic surgeries (truly or reflectively) with slopes of the same sign, Theorem 1.2 says that such genus one knots must have the same knot Floer homology as the trefoil knot. Then we use Reidemeister torsion together with number theory to get restrictions on the slopes that might appear as cosmetic surgeries. Finally, we use Heegaard Floer theory and the Casson-Walker invariant to rule out the remaining possibilities. 
The paper is organized as follows. In Section 2, we recall some preliminaries on Heegaard Floer homology. In Section 3, we establish a number theoretic result regarding cyclotomic numbers which is useful in Section 4. In Section 4, we use number theory and Heegaard Floer homology to prove Theorem 1.3.

Remark After the submission of this paper, P Ghiggini [11] proved the fibred knot conjecture for genus one knots and Y Ni [21] proved the general case. Hence our theorem could be obtained using their results without number theory.

Acknowledgements The paper was completed while the author was an exchange student at Columbia University. The author is grateful to Robion Kirby and Peter Ozsváth for their support and many helpful discussions. We thank Elisenda Grigsby, Ming-Lun Hsieh, Walter Neumann, Bjorn Poonen, Eric Urban and Shaffiq Welji for interesting discussions. Also, we would like to thank the referee for suggestions and comments on the first draft.

\section{Preliminaries in Heegaard Floer theory}

We review some of the materials and notation in Heegaard Floer theory which will be used in this article.

\subsection{Correction terms and $\operatorname{Spin}^{c}$ structures}

For a rational homology three-sphere $Y$ with $\operatorname{Spin}^{c}$ structure $\mathfrak{s}$, the minimal absolute $\mathbb{Q}$ grading of torsion-free elements in the image of $H F^{\infty}(Y, \mathfrak{s})$ in $H F^{+}(Y, \mathfrak{s})$ is an invariant of $(Y, \mathfrak{s})$, called the correction term of $(Y, \mathfrak{s})$ and denoted by $d(Y, \mathfrak{s})$. See Ozsváth and Szabó [24] for details. One basic property of the correction terms is

$$
d(Y, \mathfrak{s})=d(Y, \overline{\mathfrak{s}}), \quad d(Y, \mathfrak{s})=-d(-Y, \mathfrak{s}) .
$$

The correct term contains information of the intersection forms of four-manifolds which bound $Y$. Specifically, if $X$ is an oriented four-manifold whose intersection form is negative-definite, and $\mathfrak{t}$ is any $\operatorname{Spin}^{c}$ structure on $X$ whose restriction to $Y=\partial X$ is $\mathfrak{s}$, then we have the inequality

$$
\frac{c_{1}(\mathfrak{t})^{2}+\operatorname{rk} H^{2}(X ; \mathbb{Z})}{4} \leq d(Y, \mathfrak{s}) .
$$

In [25], Ozsváth and Szabó showed that if $X$ is a plumbed four-manifold with a negative-definite graph $G$ having at most two "bad vertices," then the correction term is a sharp bound in the above inequality. (A vertex $v$ is bad if the number of edges 
containing $v$ is bigger than minus the Euler number of the corresponding disk bundle.) Specifically, for each $\operatorname{Spin}^{c}$ structure $\mathfrak{s}$ of $Y$, the correction term $d(Y, \mathfrak{s})$ satisfies

$$
d(Y, \mathfrak{s})=\max _{K \in \operatorname{Char}(X, \mathfrak{s})} \frac{K^{2}+|G|}{4},
$$

where $|G|$ is the number of vertices of $G$, and $\operatorname{Char}(X, \mathfrak{s})$ is the set of first Chern classes of those $\operatorname{Spin}^{c}$ structures of $X$ whose restriction to $Y$ is $\mathfrak{s}$. This gives a practical way to compute the correction terms for a three-manifold $Y$ which could be realized as the boundary of such a plumbed four-manifold.

In particular, a linear plumbing with all self-intersections at most -2 has no bad vertices. In what follows, we just consider such linearly plumbed four-manifolds. A linearly plumbed four-manifold could be denoted by $\left(a_{0}, a_{1}, \cdots, a_{n}\right)\left(a_{i} \leq-2\right)$, where $\left[S_{i}\right] \cdot\left[S_{i}\right]=a_{i}$ and $\left[S_{i}\right] \cdot\left[S_{i+1}\right]=1$ (we denote by $\left[S_{i}\right]$ the homology class of the $i$-th exceptional sphere). Let $\phi_{i}$ denotes the Poincare dual of $S_{i}$. Note that if $\left(a_{0}, a_{1}, \cdots, a_{n}\right)$ gives the continued fraction expansion of $p / q$, ie,

$$
\frac{p}{q}=\left[a_{0}, a_{1}, \cdots, a_{n}\right]=a_{1}-\frac{1}{a_{2}-\frac{1}{\cdots-\frac{1}{a_{n}}}},
$$

then the boundary of the corresponding linearly plumbed four-manifold is the lens space $-L(p, q)=S_{p / q}^{3}(U)$.

Let $X$ be linearly plumbed four-manifold $\left(a_{0}, a_{1}, \cdots, a_{n}\right)$ with $a_{i} \leq-2$ and $Y$ be the boundary three-manifold of $X . Y$ is a rational homology sphere. The set of first Chern classes of $\operatorname{Spin}^{c}$ structures on $X$ is exactly the set of characteristic classes of $X$. Let $\mathfrak{s}$ be a $\operatorname{Spin}^{c}$ structure on $Y$ and $\mathfrak{t}$ be a $\operatorname{Spin}^{c}$ structure on $X$ whose restriction on $Y$ is $\mathfrak{s}$, then

$$
\operatorname{Char}(X, \mathfrak{s})=\left\{c_{1}(\mathfrak{t})+C_{0} \phi_{0}+\cdots+C_{n} \phi_{n} \mid C_{i} \text { 's are even integers }\right\} .
$$

If the first homology group of $Y$ is cyclic of an odd order, there is a unique spin $\operatorname{Spin}^{c}$ structure on $Y$, ie, the one whose first Chern class is zero. We denote this spin $\operatorname{Spin}^{c}$ structure by $\mathfrak{s}_{0}$. The set $\operatorname{Char}\left(X, \mathfrak{s}_{0}\right)$ contains those characteristic classes which could be written as a linear combination of the $\phi_{i}$ 's.

\subsection{Rational surgery formulas}

Fix a nullhomologous knot $K$ in a closed three-manifold $Y$. Knot Floer homology associates to $K$ a $\mathbb{Z} \oplus \mathbb{Z}$-filtered chain complex $C=C F K^{\infty}(Y, K)$ which is a $\mathbb{Z}[U]-$ module generated over $\mathbb{Z}$ by a set $X$. The filtration function $\mathcal{F}: X \rightarrow \mathbb{Z} \oplus \mathbb{Z}$ satisfies 
that, if $\mathcal{F}(\mathbf{x})=(i, j)$, then $\mathcal{F}(U \cdot \mathbf{x})=(i-1, j-1)$ and $\mathcal{F}(\mathbf{y}) \leq \mathcal{F}(\mathbf{x})$ for all $\mathbf{y}$ having nonzero coefficient in $\partial \mathbf{x}$.

For a subset $S$ of $\mathbb{Z} \oplus \mathbb{Z}$ with the property $(i, j) \in S$ implies $(i+1, j),(i, j+1) \in S$, let $C\{S\}$ be the quotient complex of $C$ by the subcomplex generated by $\mathbf{x}$ with $\mathcal{F}(\mathbf{x}) \notin S$. For an integer $s$, let $A_{s}^{+}(K)=C_{K}\{\max (i, j-s) \geq 0\}$ and $B^{+}(K)=C\{i \geq 0\}$. There is a canonical chain homotopy equivalence (up to sign) between $B^{+}$and $C\{j \geq 0\}$, denoted by $T$. Also, there are two chain maps $v_{s}^{+}, h_{s}^{+}: A_{s}^{+} \rightarrow B^{+}$. The map $v_{s}^{+}$is the projection of $A_{s}^{+}$onto $B^{+}$, and $h_{s}^{+}$is the composition map:

$$
A_{s}^{+} \stackrel{\text { projection }}{\longrightarrow} C\{j \geq s\} \stackrel{U^{s} .}{\longrightarrow} C\{j \geq 0\} \stackrel{T}{\longrightarrow} B^{+}
$$

We will simply write as $v^{+}$and $h^{+}$when there is no confusion.

Now fix a surgery slope $p / q$ and we suppose $q>0$. Consider the two chain complexes

$$
\mathbb{A}^{+}=\bigoplus_{t \in \mathbb{Z}}\left(t, A_{\lfloor t / q\rfloor}^{+}\right), \quad \mathbb{B}^{+}=\bigoplus_{t \in \mathbb{Z}}\left(t, B^{+}\right),
$$

where $\lfloor x\rfloor$ is the greatest integer not bigger than $x$. An element of $\mathbb{A}^{+}$could be written as $\left\{\left(t, a_{t}\right)\right\}_{t \in \mathbb{Z}}$ with $a_{t} \in A_{\lfloor t / q\rfloor}^{+}$. Define a chain map $D_{p / q}^{+}: \mathbb{A}^{+} \rightarrow \mathbb{B}^{+}$by

$$
D_{p / q}^{+}\left\{\left(t, a_{t}\right)\right\}=\left\{\left(t, b_{t}\right)\right\}, \quad b_{t}=v^{+}\left(a_{t}\right)+h^{+}\left(a_{t-p}\right) .
$$

Let $\mathbb{X}_{p / q}^{+}(K)$ be the mapping cone of $D_{p / q}^{+}$. Note that since both $A_{s}^{+}$and $B_{s}^{+}$are relatively $\mathbb{Z}$-graded, and the two chain maps $h^{+}$and $v^{+}$respect this relative grading, the chain complex $\mathbb{X}_{p / q}^{+}$can be relatively $\mathbb{Z}$-graded with the convention that the differential $D_{p / q}^{+}$lowers the grading by one.

$\mathbb{X}_{p / q}^{+}$naturally splits into the direction sum of $p$ subcomplexes

$$
\mathbb{X}_{p, q}^{+}=\bigoplus_{i=0}^{p-1} \mathbb{X}_{i, p / q}^{+},
$$

where $\mathbb{X}_{i, p / q}^{+}$is the subcomplex of $\mathbb{X}_{p / q}^{+}$containing all $A_{t}^{+}$and $B_{t}^{+}$with $t \equiv i$ $(\bmod p)$.

Ozsváth and Szabó showed that the Heegaard Floer homology of a $p / q$ surgered manifold is determined by the mapping cone $\mathbb{X}_{p / q}^{+}$.

Theorem 2.1 (Ozsváth and Szabó [23]) Let $K \in Y$ be a nullhomologous knot and $p, q$ be two coprime integers. Then there are relatively graded isomorphisms

$$
H F^{+}\left(Y_{p / q}(K)\right) \cong H_{*}\left(\mathbb{X}_{p / q}^{+}\right), \quad H F^{+}\left(Y_{p / q}(K), \mathfrak{s}_{i}\right) \cong H_{*}\left(\mathbb{X}_{i, p / q}^{+}\right),
$$

where $\mathfrak{s}_{i}$ is the $\operatorname{Spin}^{c}$ structure corresponding to $i \in \mathbb{Z} / p \mathbb{Z}$. 
In fact, the absolute grading on $\mathrm{HF}^{+}\left(Y_{p / q}(K)\right)$ is also determined. The absolute grading on $\mathbb{X}_{p / q}^{+}$is specified by fixing an absolute grading on $B_{\lfloor t / q\rfloor}^{+}$for any $t$. The absolute grading on $B_{\lfloor t / q\rfloor}^{+}$is determined by a grading on its homology $H_{*}\left(B_{\lfloor t / q\rfloor}^{+}\right) \cong$ $\mathcal{T}^{+}$, where $\mathcal{T}^{+}$is the $\mathbb{Z}[U]$-module $\mathbb{Z}\left[U, U^{-1}\right] /(U \cdot \mathbb{Z}[U])$. The absolute grading on $H_{*}\left(B_{\lfloor t / q\rfloor}^{+}\right) \cong \mathcal{T}^{+} \cong H_{*}\left(\mathbb{X}_{i, p / q}^{+}\right)$is determined so that its bottom-most nontrivial element is supported in dimension $d\left(S_{p / q}^{3}(U), i\right)=d(-L(p, q), i)$. These correction terms $d(-L(p, q), i)$ are computed by the recursive formula [24, Proposition 4.8]

$$
d(-L(p, q), i)=\left(\frac{1}{4}-\frac{(2 i+1-p-q)^{2}}{4 p q}\right)-d(-L(q, r), j),
$$

where $r \equiv p, j \equiv i(\bmod q)$ and $0 \leq r, j<q$. See [23] for details.

\section{$2.3 \quad L$-space surgeries on knots}

A rational homology three-sphere $Y$ is called an $L$-space if $\mathrm{HF}^{+}(Y)$ has no torsion and the map $H F^{\infty}(Y) \rightarrow H F^{+}(Y)$ is surjective, or equivalently, if $\widehat{H F}(Y, \mathfrak{s}) \cong \mathbb{Z}$ for each $\operatorname{Spin}^{c}$ structure $\mathfrak{s}$ on $Y$. The Heegaard Floer homology of an $L$-space is determined by its correction terms. The following proposition is implied in [23] (see [16] for results in the Seiberg-Witten gauge theory).

Proposition 2.2 Suppose $K$ is a nontrivial knot in $S^{3}$ and $S_{r}^{3}(K)$ is an $L$-space with $r>0$. Then $S_{s}^{3}(K)$ is an $L$-space for any rational number $s \geq r$.

In particular, this implies that there will be no $L$-space surgeries on a nontrivial amphichiral knot in $S^{3}$. This proposition is an ingredient to get Theorem 1.2.

\section{An equation in cyclotomic numbers}

We will need the following result regarding cyclotomic numbers in Section 4. Note that the meaning of letters in this section are different from other sections because of different conventions in number theory and topology.

Theorem 3.1 Let $m$ be an integer at least 3 and let $r$ and $r^{\prime}$ be integers such that $(r, m)=\left(r^{\prime}, m\right)=1$. Then there exists an integer $k$ such that $(k, m)=1$ and

$$
\left|\frac{\left(\xi-1+\xi^{-1}\right)}{(\xi-1)\left(\xi^{r}-1\right)}\right|=\left|\frac{\left(\xi^{k}-1+\xi^{-k}\right)}{\left(\xi^{k}-1\right)\left(\xi^{r^{\prime} k}-1\right)}\right|
$$

for any $m$-th root of unity $\xi \neq 1$ if and only if one of the following holds. 
(1) $r \equiv \pm r^{\prime}(\bmod m)$.

(2) $m=12$ and $\left\{ \pm r, \pm r^{\prime}\right\}=\{ \pm 1, \pm 5\}(\bmod 12)$.

(3) $m=18 e+9$ and $\left\{ \pm r, \pm r^{\prime}\right\}=\{ \pm(6 e+1), \pm(6 e+5)\}$ (mod $m$ ) for some nonnegative integer $e$.

Let $\zeta=\zeta_{m}=e^{2 \pi i / m}$. For $x=1,2, \cdots, m-1$, write $A_{x}=\ln \left|\zeta^{x}-1\right|$. Then when $\xi^{6} \neq 1$, we can translate equation (4) into the following multiplicative relation of cyclotomic units:

$$
\left|\frac{\left(\xi^{6}-1\right)}{\left(\xi^{2}-1\right)\left(\xi^{3}-1\right)\left(\xi^{r}-1\right)}\right|=\left|\frac{\left(\xi^{6 k}-1\right)}{\left(\xi^{2 k}-1\right)\left(\xi^{3 k}-1\right)\left(\xi^{r^{\prime} k}-1\right)}\right|
$$

In particular, when $\xi=\zeta$, in Ennola's notation, by taking the logarithm this becomes

$$
R:=A_{6}-A_{2}-A_{3}-A_{r}-\left(A_{6 k}-A_{2 k}-A_{3 k}-A_{r^{\prime} k}\right)=0 .
$$

The "if" part of the proof is easy and left to the reader. The machinery in the proof of the other direction are from Franz [9] and Dvornicich [5]. Also, when $m \leq 8$, we may compute the torsion directly to prove the theorem. So in what follows, we assume $m>8$. Since for $m \equiv 2(\bmod 4)$ there are no primitive characters, we will deal separately with the case $m \not \equiv 2(\bmod 4)$ in Section 3.2 and with the case $m \equiv 2$ $(\bmod 4)$ in Section 3.3.

\subsection{Preliminaries in number theory}

We refer to Washington [34] or Apostol [2] (which is more fundamental) for basic notation.

In 1935, Franz used the following lemma to classify lens spaces (in any dimension).

Theorem 3.2 (Franz Independence Lemma [9]) Fix a natural number $m$ and let $S=\{j \in \mathbb{Z} / m \mathbb{Z} \mid(j, m)=1\}$. Suppose that $\left\{a_{k} \mid k \in S\right\}$ is a set of integers satisfying the following.

(a) $\sum_{k \in S} a_{k}=0$

(b) $a_{j}=a_{-j}$ for all $j \in S$.

(c) $\prod_{j \in S}\left(\xi^{j}-1\right)^{a_{j}}=1$ for every $m-$ th root of unity $\xi \neq 1$.

Then $a_{j}=0$ for all $j \in S$.

Algebraic ${ }^{3} \mathcal{G}$ Geometric Topology, Volume 6 (2006) 
This lemma says that there are no multiplicative relations for the set $\left\{\zeta_{m}^{j} \mid j \in S\right\}$ where $\zeta_{m}=e^{2 \pi i / m}$. For a proof for topologists, we refer to the lecture notes of de Rham [4, Chapter 1].

For an integer $m \geq 2$. Let $\zeta=\zeta_{m}=e^{2 \pi i / m}$ and $A_{x}=\ln \left|\zeta^{x}-1\right|$ for $x \in \mathbb{Z} / m \mathbb{Z} \backslash\{0\}$. Let $R=\sum_{x=1}^{m-1} C_{x} A_{x}$ be a linear combination of $A_{x}$ with integer coefficients. A $\bmod n$ character is a multiplicative homomorphism $\chi:(\mathbb{Z} / n \mathbb{Z})^{*} \rightarrow \mathbb{C}^{*}$ and we let $\chi(i)=0$ if $(i, n)>1$. If $n \mid m$, then a $\bmod n$ character induces a mod $m$ character by composing the homomorphism $(\mathbb{Z} / n \mathbb{Z})^{*} \rightarrow(\mathbb{Z} / m \mathbb{Z})^{*}$. The conductor of a $\bmod$ $m$ character $\chi$ is the smallest $n$ such that $\chi$ is induced by a mod $n$ character. For a character $\chi(\bmod m)$ with conductor $f>1$ and integer $d \geq 1$ such that $f \mid d$ and $d \mid m$, we denote

$$
T(\chi, d, R)=\sum_{\substack{x=1 \\(x, d)=1}}^{d-1} \chi(x) C_{(m / d) x}
$$

and

$$
Y(\chi, R)=\sum_{\substack{d \\ f|d, d| m}} \frac{1}{\phi(d)} \prod_{p \mid d}(1-\bar{\chi}(p)) T(\chi, d, R) .
$$

For $p \mid m$, define $\gamma_{p}$ by $p^{\gamma_{p}} \mid m$ and $p^{\gamma_{p}+1} \nmid m$ and denote

$$
Y_{p}(R)=\sum_{x=1}^{p^{\gamma p}-1}\left(x, p^{\gamma_{p}}\right) C_{\left(m / p^{\gamma p}\right) x}
$$

Ennola gave the following characterization of relations in cyclotomic numbers:

Theorem 3.3 (Ennola [7]) With all notation as above, $R=0$ if and only if the following two conditions hold:

$$
\begin{aligned}
Y(\chi, R)=0 & \text { for every even character } \chi \neq \chi_{1} \\
Y_{p}(R)=0 & \text { for every prime number } p \text { dividing } m
\end{aligned}
$$

where $\chi_{1}$ is the principle character defined by $\chi_{1}(x)=1$ for $(x, m)=1$ (and $\chi_{1}(x)=0$ if $(x, m)>1)$.

In our application of this theorem, the second relations that $Y_{p}(R)=0$ for every prime number $p$ dividing $m$ are automatically satisfied. We shall prove our results as consequence of the relations (7).

Dvornicich gave the following propositions about characters (in order to solve his equation in cyclotomic numbers): 
Proposition 3.4 (Dvornicich [5, Corollary 1]) Let $m \geq 2$. If $k$ satisfies $\chi(k)=1$ for all even primitive characters of a maximal conductor $C$, where $C=m / 2$ if $m \equiv 2$ $(\bmod 4)$ and $C=m$ otherwise, then

(a) $k \equiv \pm 1, \pm i(\bmod m)$ if $m=2^{a} \cdot 3, a \geq 3$, where $i=1-2^{a-1}$ if $a$ is even and $i=1+2^{a-1}$ when $a$ is odd.

(b) $k \equiv \pm 1(\bmod m / 2)$ if $m \equiv 2(\bmod 4)$.

(c) $k \equiv \pm 1(\bmod m)$ otherwise.

Proposition 3.5 (Dvornicich [5, Lemma 3]) If $m \geq 3$ is odd. Let $G$ be the group of all even Dirichlet characters mod $m$. Then the set of Dirichlet even characters of maximal conductor is not contained in the union of two maximal subgroups of $G$, unless $m=3 m_{1} m_{2}$ with $m_{1}, m_{2}>1$ and $\left(3, m_{1}\right)=\left(3, m_{2}\right)=\left(m_{1}, m_{2}\right)=1$.

The following lemma is told to me by Bjorn Poonen in private communication. This lemma says that if four roots of unity add up to zero, then they must cancel in pairs.

Lemma 3.6 Suppose $\xi_{i}, 1 \leq i \leq 4$, are all roots of unity and $\xi_{1}+\xi_{2}=\xi_{3}+\xi_{4}$. Then $\xi_{1}+\xi_{2}=0, \xi_{1}=\xi_{3}$ or $\xi_{1}=\xi_{4}$.

Proof Suppose that $\xi_{1}+\xi_{2} \neq 0$. Considering the angle and norm of $\xi_{1}+\xi_{2}$ and $\xi_{3}+\xi_{4}$ will give the lemma.

\subsection{Proof of theorem when $m \not \equiv 2(\bmod 4)$}

Pick an integer $k^{\prime}$ such that $r^{\prime} \equiv r k^{\prime}(\bmod m)$.

Case $1(m, 6)=1$.

In this case, we have $\xi^{6} \neq 1$ for all $m$-th roots of unity $\xi \neq 1$. Hence equation (5) holds for all $m$-th roots of unity $\xi \neq 1$ and is nonzero. So the Franz Independence Lemma applies and we get

$$
\left\{ \pm 6, \pm 2 k, \pm 3 k, \pm r^{\prime} k\right\}=\{ \pm 6 k, \pm 2, \pm 3, \pm r\} \quad(\bmod m) .
$$

By congruence arguments, we can show that $r \equiv \pm r^{\prime}(\bmod m)$.

Case $2(m, 12)=4$.

Let $b$ be the $\bmod m$ solution of the congruence equation $r \equiv 3 b k(\bmod m)$. Consider all relations (7) relative to characters of maximal conductor. They have the form

$$
\chi(3)+\chi(r)-\chi(3 k)-\chi\left(r k^{\prime} k\right)=0 .
$$

(All other terms are zero since they are even and not coprime to $m$.) By Lemma 3.6 we have the following three possibilities. 
(1) $\chi(3)+\chi(r)=\chi(3 k)+\chi\left(r k^{\prime} k\right)=0$. Since $\chi(k) \neq 0$, it follows that $\chi(r)=$ $\chi\left(r k^{\prime}\right)$ and hence $\chi\left(k^{\prime}\right)=1$.

(2) $\chi(3)-\chi(3 k)=\chi(r)-\chi\left(r k^{\prime} k\right)=0$. We get $\chi(k)=1$ and hence $\chi\left(k^{\prime}\right)=1$.

(3) $\chi(3)-\chi\left(r k^{\prime} k\right)=\chi(r)-\chi(3 k)=0$. So $\chi(3 b k)-\chi(3 k)=0$ and $\chi(b)=1$.

In all cases, we get

$$
\left(\chi\left(k^{\prime}\right)-1\right)(\chi(b)-1)=0 .
$$

Suppose $k^{\prime} \not \equiv \pm 1, b \not \equiv \pm 1(\bmod m)$. Let $G_{1}, G_{2}$ be the subgroup of $G$ defined by $\chi\left(k^{\prime}\right)=1$ and $\chi(b)=1$. By Proposition 3.4, $G_{1}, G_{2}$ are proper subgroups of $G$. Now by Proposition 3.5, we have the equalities $m=3 m_{1} m_{2}$ with $m_{1}, m_{2}>1$ and $\left(3, m_{1}\right)=\left(3, m_{2}\right)=\left(m_{1}, m_{2}\right)=1$, which is impossible since $(m, 3)=1$. Therefore either $k^{\prime} \equiv \pm 1(\bmod m)$ or $b \equiv \pm 1(\bmod m)$.

If $k^{\prime} \equiv \pm 1(\bmod m)$, we then have $r \equiv \pm r^{\prime}(\bmod m)$.

If $b \equiv \pm 1(\bmod m)$, then $r \equiv \pm 3 k(\bmod m)$ and our equation $(6)$ is reduced to

$$
A_{6}-A_{2}-A_{3}-A_{6 k}+A_{2 k}+A_{r^{\prime} k}=0 .
$$

Now for any even character of maximal conductor, (7) becomes

$$
-\chi(3)+\chi\left(r^{\prime} k\right)=0 \text {. }
$$

Again by Proposition 3.4, we get $r^{\prime} k \equiv \pm 3(\bmod m)$. This reduces our equation further to

$$
A_{6}-A_{2}-A_{6 k}+A_{2 k}=0 .
$$

Rewrite this equation to get

$$
\left|\zeta^{2}+1+\zeta^{-2}\right|=\left|\zeta^{2 k}+1+\zeta^{-2 k}\right| \text { and }|1+2 \cos (4 \pi / m)|=|1+2 \cos (4 k \pi / m)| \text {. }
$$

Note that since $m>8$, we have $\cos (4 \pi / m)>0$; hence $\cos (4 k \pi / m)>0$ and we have

$$
\zeta^{2}+1+\zeta^{-2}=\zeta^{2 k}+1+\zeta^{-2 k}
$$

By Lemma 3.6, $2 k \equiv \pm 2(\bmod m)$. So either $k \equiv \pm 1(\bmod m)$ or $k \equiv m_{1} \pm 1$ $(\bmod m)$, where $m_{1}=m / 2$. If $k \equiv \pm 1(\bmod m)$, we get $r= \pm 3, r^{\prime}= \pm 3(\bmod m)$. If $k \equiv m_{1} \pm 1(\bmod m)$, then $r \equiv \pm\left(m_{1}+3\right)$ and $r^{\prime} \equiv \pm\left(m_{1}+3\right) \quad(\bmod m)$. Therefore $r \equiv \pm r^{\prime}(\bmod m)$.

Case $3(m, 6)=3$.

Let $b$ be the $\bmod m$ solution of the congruence equation $r \equiv 2 b k(\bmod m)$. Consider all relations (7) relative to characters of maximal conductor. They have the form

$$
\chi(2)+\chi(r)-\chi(2 k)-\chi\left(r k^{\prime} k\right)=0 .
$$


By Lemma 3.6, we have the following three possibilities.

(1) $\chi(2)+\chi(r)=\chi(2 k)+\chi\left(r k^{\prime} k\right)=0$. Hence $\chi(k)\left(\chi(2)+\chi\left(r k^{\prime}\right)\right)=0$ and it follows that $\chi(r)=\chi\left(r k^{\prime}\right)$ and therefore $\chi\left(k^{\prime}\right)=1$.

(2) $\chi(2)-\chi(2 k)=\chi(r)-\chi\left(r k^{\prime} k\right)=0$. We get $\chi(k)=1$ and hence $\chi\left(k^{\prime}\right)=1$.

(3) $\chi(2)-\chi\left(r k^{\prime} k\right)=\chi(r)-\chi(2 k)=0$. So $\chi(2 b k)-\chi(2 k)=0$ and $\chi(b)=1$.

In all three cases, we get

$$
\left(\chi\left(k^{\prime}\right)-1\right)(\chi(b)-1)=0 .
$$

Suppose $k^{\prime} \not \equiv \pm 1, b \not \equiv \pm 1(\bmod m)$. Let $G_{1}, G_{2}$ be the subgroup of $G$ defined by $\chi\left(k^{\prime}\right)=1$ and $\chi(b)=1$. By Proposition 3.4, $G_{1}, G_{2}$ are proper subgroups of $G$. Thus we have $m=3 m_{1} m_{2}$ with $m_{1}, m_{2}>1$ and $\left(3, m_{1}\right)=\left(3, m_{2}\right)=\left(m_{1}, m_{2}\right)=1$ by Proposition 3.5 .

So there are three possibilities.

(1) If $k^{\prime} \equiv \pm 1(\bmod m)$, we get $r \equiv \pm r^{\prime}(\bmod m)$.

(2) If $b \equiv \pm 1(\bmod m)$, ie, $r \equiv \pm 2 k(\bmod m)$, equation (6) is reduced to

$$
A_{6}-A_{2}-A_{3}-A_{6 k}+A_{3 k}+A_{r^{\prime} k}=0 .
$$

Now for any even character of maximal conductor, (7) becomes

$$
-\chi(2)+\chi\left(r^{\prime} k\right)=0 .
$$

Again by Proposition 3.4, we get $r^{\prime} k \equiv \pm 2(\bmod m)$. This reduces our equation further to

$$
A_{6}-A_{3}-A_{6 k}+A_{3 k}=0 .
$$

This gives $\left|\zeta^{3}+1\right|=\left|\zeta^{3 k}+1\right|$. Hence $3 k \equiv \pm 3(\bmod m)$ and we get the congruence system

$$
3 k \equiv \pm 3, \quad r \equiv \pm 2 k, \quad r^{\prime} k \equiv \pm 2 \quad(\bmod m)
$$

Using congruence arguments, we can show that either $r \equiv \pm r^{\prime}(\bmod m)$, or, for some nonnegative integer $e$,

$$
m=18 e+9 \quad \text { and } \quad\left\{ \pm r, \pm r^{\prime}\right\}=\{ \pm(6 e+1), \pm(6 e+5)\} .
$$

(3) If $m=3 m_{1} m_{2}$ with $m_{1}, m_{2}>1$ and $\left(3, m_{1}\right)=\left(3, m_{2}\right)=\left(m_{1}, m_{2}\right)=1$. In equation (5), let $\xi=\zeta_{m}^{3 a}$ with $m_{1} m_{2} \nmid a$, and we find that equation (5) holds for all $m_{1} m_{2}$-th root of unity $\xi \neq 1$ and $\left(m_{1} m_{2}, 6\right)=1$. So by case 1 , we get $r \equiv \pm r^{\prime}$ 
$\left(\bmod m_{1} m_{2}\right)$ and $k \equiv \pm 1\left(\bmod m_{1} m_{2}\right)$. This implies $3 k \equiv \pm 3,6 k \equiv \pm 6(\bmod m)$. So equation (5) reduces to

$$
\left|\left(\xi^{2}-1\right)\left(\xi^{r}-1\right)\right|=\left|\left(\xi^{2 k}-1\right)\left(\xi^{r^{\prime} k}-1\right)\right| .
$$

Now the Franz Independence Lemma applies and we get

$$
\{ \pm 2, \pm r\}=\left\{ \pm 2 k, \pm r^{\prime} k\right\} \quad \text { and } 3 k \equiv \pm 3 \quad(\bmod m) .
$$

If $2 k \equiv \pm 2(\bmod m)$, then we have $k \equiv \pm 1, \pm 5(\bmod m)$. If $k \equiv \pm 5(\bmod m)$, we get $10 \equiv \pm 2,15 \equiv \pm 3(\bmod m)$, which implies $m=12$ and which is impossible since $m$ is odd. So we have $k \equiv \pm 1(\bmod m)$ and $r \equiv \pm r^{\prime}(\bmod m)$.

If $2 k \equiv \pm r(\bmod m)$, then we get the same congruence system (10). So $r \equiv \pm r^{\prime}$ $(\bmod m)$ since $m$ can not be of the form $18 e+9$.

This ends the proof of the theorem in Case 3.

Case $4(m, 12)=12$.

For all characters of maximal conductor, Ennola's relations (7) have the form

$$
\chi(r)-\chi\left(r^{\prime} k\right)=0, \quad \text { ie, } \quad \chi(r)-\chi\left(r k^{\prime} k\right)=0 .
$$

So we get $\chi\left(k^{\prime} k\right)=1$. Now by Proposition 3.4, we have one of the following.

(1) $k^{\prime} k \equiv \pm 1$ if $m$ is not of the form $2^{a} \cdot 3, a \geq 3$.

(2) $k^{\prime} k \equiv \pm 1, \pm i(\bmod m)$ if $m=2^{a} \cdot 3, a \geq 3$. Here $i=1-2^{a-1}$ if $2 \mid a$ and $i=1+2^{a-1}$ if $2 \nmid a$.

Subcase 4.1 $m \neq 2^{a} \cdot 3$ for any $a \geq 3$.

In this subcase, we will get $r \equiv \pm r^{\prime} k(\bmod m)$. So our equation (6) reduces to

$$
R^{\prime}:=A_{6}-A_{2}-A_{3}-\left(A_{6 k}-A_{2 k}-A_{3 k}\right)=0 .
$$

Write $m=12 m_{1}$. Consider even characters $\chi$ of conductor $f_{1}=4 m_{1}$.

Let $d$ be an integer such that $f_{1}|d| m$. Since $m / f_{1}=3, m / d=1,3$. Let $d_{1}=12 m_{1}$ and $d_{2}=4 m_{1}$. For $d_{1}=12 m_{1}$, we have

$$
T\left(\chi, d_{1}, R^{\prime}\right)=\sum_{\substack{x=1 \\(x, m)=1}}^{m-1} \chi(x) C_{x}=0
$$

since $(x, m)>1$ for every $C_{x} \neq 0$. For $d_{2}$, we get

$$
T\left(\chi, d_{2}, R^{\prime}\right)=-\chi(1)+\chi(k)=\chi(k)-1 .
$$

Algebraic 83 Geometric Topology, Volume 6 (2006) 
So (7) for such characters becomes

$$
\frac{1}{\varphi\left(f_{1}\right)}(\chi(k)-1)=0 .
$$

So $\chi(k)=1$ and by Proposition 3.4 we get $k \equiv \pm 1 \bmod f_{1}$, or $k \equiv \pm i\left(\bmod f_{1}\right)$ if $f_{1}=2^{a} \cdot 3, a \geq 3$ (equivalently $m=2^{a} \cdot 9$ ).

If $k \equiv \pm i\left(\bmod f_{1}\right)$, then $m=2^{a} \cdot 9, a \geq 3$, and we have

$$
\begin{array}{lll}
k \equiv \pm\left(1-2^{a-1}\right) & \left(\bmod 2^{a}\right) & \text { if } 2 \mid a \\
k \equiv \pm\left(1+2^{a-1}\right) & \left(\bmod 2^{a}\right) & \text { if } 2 \nmid a .
\end{array}
$$

For any even character $\chi$ of conductor $f=2^{a}$, we have $m / f=9$ and $d$ could be $d_{1}=m, d_{2}=m / 3$ and $d_{3}=m / 9$. For our equation (11), we have

$$
\begin{gathered}
T\left(\chi, d_{1}, R^{\prime}\right)=\sum_{\substack{x=1 \\
(x, m)=0}}^{m-1} \chi(x) C_{x}=0, \quad T\left(\chi, d_{3}, R^{\prime}\right)=\sum_{\substack{x=1 \\
\left(x, 2^{a}\right)=0}}^{d_{3}-1} \chi(x) C_{9 x}=0, \\
T\left(\chi, d_{2}, R^{\prime}\right)=\sum_{\substack{x=1 \\
\left(x, 2^{a} \cdot 3\right)=0}}^{d_{2}-1} \chi(x) C_{3 x}=-\chi(1)+\chi(k) .
\end{gathered}
$$

So $Y\left(\chi, R^{\prime}\right)=0$ gives $-\chi(1)+\chi(k)=0$. By Proposition 3.4 , we get $k \equiv \pm 1$ $\left(\bmod 2^{a}\right)$. This is a contradiction to (12) since $a \geq 3$. So we must have $k \equiv \pm 1$ $\left(\bmod f_{1}\right)$.

If $k \equiv \pm 1\left(\bmod f_{1}\right)$, then we have $6 k \equiv \pm 6,3 k \equiv \pm 3(\bmod m)$. And equation (11) implies $A_{2}=A_{2 k}$. So we have the system

$$
\left\{\begin{array}{l}
2 k \equiv \pm 2 \quad(\bmod m) \\
3 k \equiv \pm 3 \quad(\bmod m)
\end{array}\right.
$$

So $k \equiv \pm 1, \pm 5(\bmod m)$. If $k \equiv \pm 1(\bmod m)$, then $k^{\prime} \equiv \pm 1(\bmod m)$ and hence $r \equiv \pm r^{\prime}(\bmod m)$. If $k \equiv \pm 5(\bmod m)$, then $10 \equiv \pm 2(\bmod m)$ and hence $m=12$ since $12 \mid m$. By an easy computation, we can conclude $\left\{ \pm r, \pm r^{\prime}\right\}=\{ \pm 1, \pm 5\}$.

This ends the proof of theorem in subcase 4.1.

Subcase $4.2 m=2^{a} \cdot 3, a \geq 3$.

Let us first suppose $k^{\prime} k \equiv \pm i(\bmod m)$. If $a$ is even, then we get $k^{\prime} k \equiv \pm\left(1-2^{a-1}\right)$ $(\bmod m)$. If $a$ is odd, then $k^{\prime} k \equiv \pm\left(1+2^{a-1}\right)(\bmod m)$. 
Let $\chi_{0}$ be a generator of the even characters $\bmod 2^{a}$; we have $\chi_{0}\left( \pm k^{\prime} k\right)=-1$ and the relation $Y\left(\chi_{0}, R\right)=0$ becomes

$$
\frac{1}{2^{a-1}} \sum_{\substack{x=1 \\\left(x, 2^{a}\right)=1}}^{2^{a}-1} \chi_{0}(x) C_{3 x}+\frac{1}{2 \cdot 2^{a-1}}\left(1-\bar{\chi}_{0}(3)\right) \sum_{\substack{x=1 \\\left(x, 2^{a} \cdot 3\right)=1}}^{2^{a} \cdot 3-1} \chi_{0}(x) C_{x}=0 .
$$

And for our relation $R$, we read

$$
2\left(-1+\chi_{0}(k)\right)+\left(1-\bar{\chi}_{0}(3)\right)\left(-\chi_{0}(r)+\chi_{0}\left(r k^{\prime} k\right)\right)=0 .
$$

Since $\chi_{0}\left(k^{\prime} k\right)=-1$, we get

$$
\chi_{0}(k)-1=\chi_{0}(r)\left(1-\bar{\chi}_{0}(3)\right) .
$$

This gives a 4 -term relation among roots of unity. By Lemma 3.6, since $\chi_{0}(3) \neq 1$, we have the following two possibilities.

(1) $\chi_{0}(k)=\chi_{0}(r), 1=\chi_{0}(r) \bar{\chi}_{0}(3)$. Hence we have $\chi_{0}(k)=\chi_{0}(r)=\chi_{0}(3)$.

(2) $\chi_{0}(k)=-\chi_{0}(r) \bar{\chi}_{0}(3), \chi_{0}(r)=-1$. Let $\bar{k}, \bar{k}^{\prime}$ be integers where $\bar{k}^{\prime} k^{\prime} \equiv 1$ and $\bar{k} k \equiv 1(\bmod m)$. Using $\chi_{0}\left(k^{\prime} k\right)=-1$, we may conclude that these two equations will be equivalent to $\chi_{0}(\bar{k})=\chi_{0}\left(\bar{k}^{\prime}\right)=\chi_{0}(3)$.

By symmetry, we may just consider one possibility. Suppose $\chi_{0}(k)=\chi_{0}(r)=\chi_{0}(3)$.

Consider the character $\chi_{1}=\chi_{0}^{2}$. It is a character of conductor $2^{a-1}=m / 6$. There are only four possibilities: $d_{1}=m, d_{2}=m / 2=3 \cdot 2^{a-1}, d_{3}=m / 3=2^{a}$ and $d_{4}=m / 6=2^{a-1}$. Since $\chi_{0}\left(k^{\prime} k\right)=-1$ and $\chi_{0}(k)=\chi(3)$, we have

$$
\begin{aligned}
& T\left(\chi_{1}, d_{1}, R\right)=-\chi_{1}(r)+\chi_{1}\left(r k^{\prime} k\right)=\chi_{1}(r)\left(\chi_{0}^{2}\left(k^{\prime} k\right)-1\right)=0, \\
& T\left(\chi_{1}, d_{2}, R\right)=\sum_{\substack{x=1 \\
\left(x, 3 \cdot 2^{a-1}\right)=1}}^{d_{2}-1} \chi_{1}(x) C_{2 x}=-\chi_{1}(1)+\chi_{1}(k)=\chi_{0}^{2}(3)-1, \\
& T\left(\chi_{1}, d_{3}, R\right)=\chi_{0}^{2}(3)-1 \text { and } T\left(\chi_{1}, d_{4}, R\right)=1-\chi_{0}^{2}(3) .
\end{aligned}
$$

Therefore, since $\chi_{1}(2)=0$, the relation (7) for the character $\chi_{1}$ becomes

$$
\begin{aligned}
Y\left(\chi_{1}, R\right) & =\frac{1}{2 \cdot 2^{a-2}}\left(1-\bar{\chi}_{0}^{2}(3)\right)\left(\chi_{0}^{2}(3)-1\right)+\frac{1}{2^{a-1}}\left(\chi_{0}^{2}(3)-1\right)+\frac{1}{2^{a-2}}\left(1-\chi_{0}^{2}(3)\right) \\
& =\frac{\left(\chi_{0}^{2}(3)-1\right)}{2^{a-1}}\left(\left(1-\bar{\chi}_{0}^{2}(3)\right)+1-2\right)=\frac{\bar{\chi}_{0}^{2}(3)\left(\chi_{0}^{2}(3)-1\right)}{2^{a-1}} .
\end{aligned}
$$


For $a=3$, we may prove the theorem by direct computation. And when $\chi_{0}^{2}(3) \neq 1$, this is a contradiction. Hence $k^{\prime} k \equiv \pm 1(\bmod m)$.

When $k^{\prime} k \equiv \pm 1(\bmod m)$, we reduce our equation to

$$
R^{\prime}:=A_{6}-A_{2}-A_{3}-\left(A_{6 k}-A_{2 k}-A_{3 k}\right)=0 .
$$

For the character $\chi_{0}$, we get

$$
Y\left(\chi_{0}, R^{\prime}\right)=\frac{1}{2^{a-1}}\left(-1+\chi_{0}(k)\right)=0,
$$

and hence $\chi_{0}(k)=1$. Therefore $k \equiv \pm 1\left(\bmod 2^{a}\right)$. This implies $3 k \equiv \pm 3,6 k \equiv \pm 6$ $(\bmod m)$ and $R^{\prime}=0$ will reduce to $A_{2}=A_{2 k}$. So we get $2 k \equiv \pm 2, \pm(m / 2+2)$ $(\bmod m)$. Combining $k \equiv \pm 1\left(\bmod 2^{a}\right)$, we get $k \equiv \pm 1(\bmod m)$. Hence $k^{\prime} \equiv \pm 1$ $(\bmod m)$, which implies $r \equiv \pm r^{\prime}(\bmod m)$. This ends the proof of the theorem in subcase 4.2 .

\subsection{Proof of theorem when $m \equiv 2(\bmod 4)$}

There is no primitive character with modulus $m$. Write $m=2 m_{1}$, where $m_{1}=2 a+1$ is odd. For any $m_{1}-$ th root of unity $\xi,-\xi$ is a $m$-th root of unity, hence we have

$$
\left|\frac{\xi+1+\xi^{-1}}{(\xi+1)\left(\xi^{r}+1\right)}\right|=\left|\frac{\xi^{k}+1+\xi^{-k}}{\left(\xi^{k}+1\right)\left(\xi^{r^{\prime} k}+1\right)}\right| .
$$

When $\xi \neq 1$, this could be transformed into

$$
\left|\frac{\left(\xi^{3}-1\right)\left(\xi^{r}-1\right)}{\left(\xi^{2}-1\right)\left(\xi^{2 r}-1\right)}\right|=\left|\frac{\left(\xi^{3 k}-1\right)\left(\xi^{r^{\prime} k}-1\right)}{\left(\xi^{2 k}-1\right)\left(\xi^{2 r^{\prime} k}-1\right)}\right| .
$$

In particular, this holds for $\xi=\zeta_{m_{1}}$. So in Ennola's notation, we have the mod $m_{1}$ relation

$$
R_{1}:=A_{3}+A_{r}-A_{2}-A_{2 r}-\left(A_{3 k}+A_{r^{\prime} k}-A_{2 k}-A_{2 r^{\prime} k}\right)=0 .
$$

Note that $r, k, r^{\prime}$ are all coprime to 2 and $m_{1}$.

Case $1 \quad\left(m_{1}, 3\right)=1$.

In this case, equation (14) is

$$
\left|\frac{\left(\xi^{3}-1\right)\left(\xi^{r}-1\right)}{\left(\xi^{2}-1\right)\left(\xi^{2 r}-1\right)}\right|=\left|\frac{\left(\xi^{3 k}-1\right)\left(\xi^{r^{\prime} k}-1\right)}{\left(\xi^{2 k}-1\right)\left(\xi^{2 r^{\prime} k}-1\right)}\right| \neq 0,
$$

Algebraic $8 \mathcal{G}$ Geometric Topology, Volume 6 (2006) 
and holds for any $m_{1}$-th root of unity $\xi \neq 1$. So the Franz Independence Lemma implies that

$$
\left\{ \pm 3, \pm r, \pm 2 k, \pm 2 r^{\prime} k\right\}=\left\{ \pm 3 k, \pm r^{\prime} k, \pm 2, \pm 2 r\right\} \quad\left(\bmod m_{1}\right)
$$

By a congruence argument, we get $r \equiv \pm r^{\prime}\left(\bmod m_{1}\right)$ or

$$
r \equiv \pm 2, k \equiv \pm 2, r^{\prime} \equiv \pm \frac{m_{1}+3}{2} \quad\left(\bmod m_{1}\right)
$$

or a symmetric case. In the first case, we get $r \equiv \pm r^{\prime}(\bmod m)$ since $r \equiv \pm r^{\prime}$ $(\bmod 2)$. In the second case, we get $r \equiv \pm k$ and $r^{\prime} k \equiv \pm 3\left(\bmod m_{1}\right)$. Note that equation (4) also holds for any $m_{1}$-th root $\xi$, so we have

$$
\left|\frac{\xi-1+\xi^{-1}}{\xi-1}\right|=\left|\frac{\xi^{2}-1+\xi^{-2}}{\xi^{3}-1}\right| .
$$

When $\xi \neq-1$, this could be simplified as $\left|\xi^{2}+1+\xi^{-2}\right|=\left|\xi^{2}-1+\xi^{-2}\right|$, or equivalently, $\left|2 \operatorname{Re}\left(\xi^{2}\right)+1\right|=\left|2 \operatorname{Re}\left(\xi^{2}\right)-1\right|$. We hence get $\operatorname{Re}\left(\xi^{2}\right)=0$ for any $m_{1}-$ th root of unity other than \pm 1 , which is a contradiction. So we must have $r \equiv \pm r^{\prime}(\bmod m)$.

Case $2\left(m_{1}, 3\right)=3$.

For any even character $\chi$ of maximal conductor, (7) has the form

$$
\chi(r)-\chi(2)-\chi(2 r)-\chi\left(r^{\prime} k\right)+\chi(2 k)+\chi\left(2 r^{\prime} k\right)=0 .
$$

In equation (5), let $\xi=\zeta_{m}^{2}=\zeta_{m_{1}}$. Then no term in equation (5) will be zero (note that we suppose $m>8$ ). And we will get equation (6) as a mod $m_{1}$ relation. So for any even character $\chi$ of maximal conductor, we get

$$
-\chi(2)-\chi(r)+\chi(2 k)+\chi\left(r^{\prime} k\right)=0 .
$$

Substitute $\chi\left(r^{\prime} k\right)$ in $(15)$ by $\chi(2)+\chi(r)-\chi(2 k)$ to get

$$
\chi(2)(2-\chi(2))(\chi(k)-1)=0 \text {. }
$$

Hence $\chi(k)=1$ and by Proposition 3.4 , we get $k \equiv \pm 1\left(\bmod m_{1}\right)$. Since $(m, k)=1$, we conclude that $k \equiv \pm 1(\bmod m)$. Now equation (13) reduces to

$$
\left|\xi^{r}+1\right|=\left|\xi^{r^{\prime}}+1\right|
$$

and we get $r \equiv \pm r^{\prime}(\bmod m)$.

This ends the proof of our theorem. 


\section{Cosmetic surgeries on genus one knots}

In this section, we prove Theorem 1.3.

Our focus will be on knots having the same knot Floer homology as the right-handed trefoil. For a knot $K$ in $S^{3}$, we call any knot having the same knot Floer homology as $K$ a fake $K$ knot. In particular, $K$ is itself a fake $K$ knot.

Using the notation of Section 2, for any fake right-handed trefoil knot $K$, we have $H_{*}\left(A_{s}^{+}\right) \cong H_{*}\left(B^{+}\right) \cong \mathcal{T}^{+}$, where $\mathcal{T}^{+}$is the $\mathbb{Z}[U]$-module $\mathbb{Z}\left[U, U^{-1}\right] /(U \cdot \mathbb{Z}[U])$. The two maps on homology (still denoted by $h^{+}$and $v^{+}$) induced by $h^{+}: A_{s}^{+} \rightarrow B^{+}$ and $v^{+}: A_{s}^{+} \rightarrow B^{+}$are identified with multiplication by powers of $U$ as follows.

$$
h_{s}^{+}=\left\{\begin{array}{ll}
1 \cdot & \text { if } s<0 \\
U \cdot & \text { if } s=0 \\
U^{s} . & \text { if } s>0
\end{array} \quad v_{s}^{+}= \begin{cases}1 \cdot & \text { if } s>0 \\
U \cdot & \text { if } s=0 \\
U^{-s} . & \text { if } s<0\end{cases}\right.
$$

Observe that $h_{s}^{+}=v_{-s}^{+}$, and we get a symmetry of the chain complex $\mathbb{X}_{p / q}^{+}$given by

$$
(t, x) \longleftrightarrow(q-t-1, x), \quad x \in \mathcal{T}^{+} .
$$

As a motivational application of the rational surgery (to our propositions), we give the chain complex $\mathbb{X}_{5 / 3}^{+}(K)$ illustrated as follows.

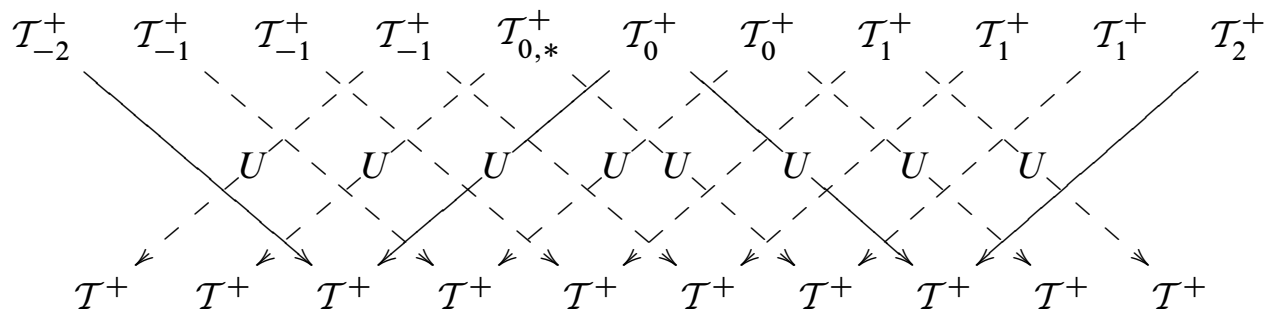

In this diagram, the one with a star subscript corresponds to $\left(0, A_{0}^{+}\right)$, while the number in the subscript means that $\mathcal{T}_{i}^{+}$corresponds to $A_{i}^{+}$. Also the right arrows denote $h^{+}$ while the left arrows denote $v^{+}$. The label at the middle of an arrow gives the map in the sense of (16), and those unlabeled ones are identities.

Algebraic 8 Geometric Topology, Volume 6 (2006) 
Similarly, the chain complex $\mathbb{X}_{5 / 4}^{+}(K)$ could be illustrated as follows.

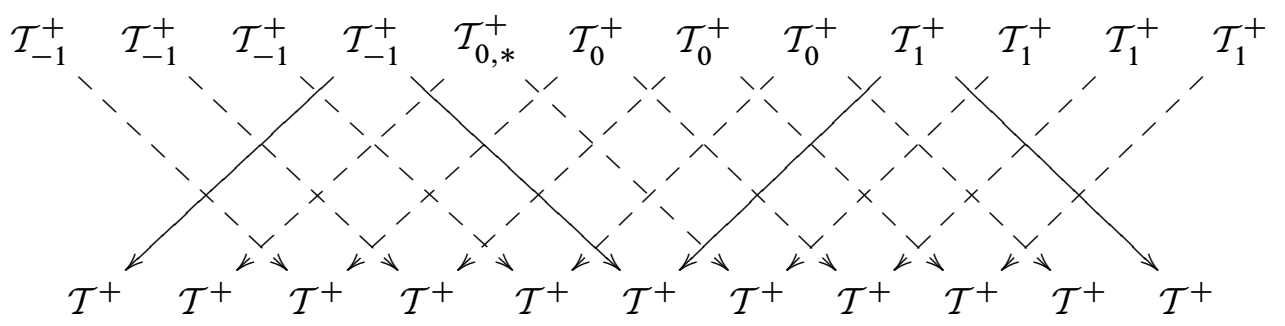

Here we omit the illustration of homomorphisms, but one could identify them using formula (16).

We now establish some propositions on the Heegaard Floer homology of a surgered manifold on a fake right-handed trefoil knot.

Proposition 4.1 Suppose $K$ is a fake right-handed trefoil knot, then no negative surgery on $K$ yields an $L$-space. And symmetrically, there are no positive surgeries on fake left-handed trefoil knots yielding $L$-spaces.

Proof This is an application of the rational surgery formula (Theorem 2.1).

Proposition 4.2 For any nontrivial knot $K$ in $S^{3}$, if $S_{r}^{3}(K)$ is an $L$-space, then $|r| \geq 1$.

Proof This is a direct consequence of the rank formula of $\widehat{H F}\left(S_{r}^{3}(K)\right)$ [23, Proposition 9.5].

Proposition 4.3 Let $p$ and $q$ be a pair of positive coprime integers. Suppose that $p$ is odd and $q \leq p$. Let $U$ be the unknot and $K$ be a fake right-handed trefoil knot, then, for the spin $\operatorname{Spin}^{c}$ structure $\mathfrak{s}_{0}$, we have

$$
d\left(S_{p / q}^{3}(K), \mathfrak{s}_{0}\right)= \begin{cases}d\left(S_{p / q}^{3}(U), \mathfrak{s}_{0}\right), & \text { if } q \text { is even }, \\ d\left(S_{p / q}^{3}(U), \mathfrak{s}_{0}\right)-2, & \text { if } q \text { is odd } .\end{cases}
$$

Proof We apply the rational surgery formula for Heegaard Floer homology (Theorem 2.1) here.

In view of the property of the correction terms (1), the symmetry mentioned above implies that the central subcomplex which is preserved under the symmetry (17) has the same homology as that corresponding to the spin $\operatorname{Spin}^{c}$ structure $\mathfrak{s}_{0}$. (In the diagrams 
illustrating the chain complexes for $S_{5 / 3}^{3}(T)$ and $S_{5 / 4}^{3}(T)$, the two central complexes are represented by solid lines.)

When $q$ is odd (see the complex for $S_{5 / 3}^{3}(K)$ as an example), this central subcomplex has the following form:

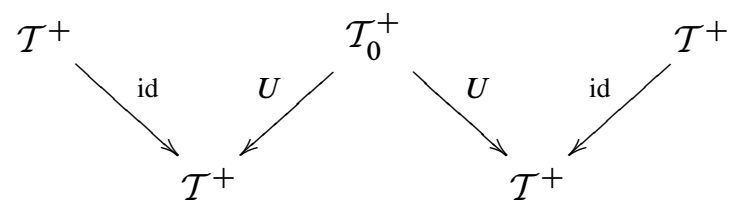

From the absolute grading determination, we see immediately that

$$
d\left(S_{p / q}^{3}(K), \mathfrak{s}_{0}\right)=d\left(S_{p / q}^{3}(U), \mathfrak{s}_{0}\right)-2,
$$

since the lowest degree term in $\mathcal{T}_{0}^{+} \cong H_{*}\left(A_{0}^{+}\right)$is killed by multiplication with $U$ and whose degree is 2 lower than that of the element mapping to the lowest degree element in $\mathcal{T}^{+} \cong H_{*}\left(B^{+}\right)$.

When $q$ is even (see the complex for $S_{5 / 4}^{3}(K)$ as an example), the central subcomplex has the form

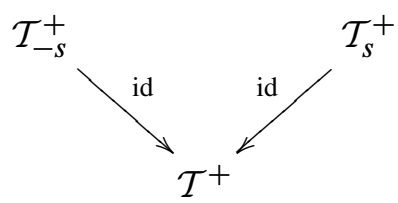

where $s=\lfloor(p+q+1) /(2 q)\rfloor$. Note that here we need the condition $q \leq p$ so that the two maps above are identities. So by the absolute grading determination, we obtain

$$
d\left(S_{p / q}^{3}(K), \mathfrak{s}_{0}\right)=d\left(S_{p / q}^{3}(U), \mathfrak{s}_{0}\right)
$$

Remark Proposition 4.3 could also be obtained by a surgery formula on correction terms given by Rustamov [30, Proposition 3.1]. Note that the spin $\mathrm{Spin}^{c}$ structure $\mathfrak{s}_{0}$ corresponds to $l \equiv(p-1)(1-x) / 2(\bmod p)$, where $q x \equiv-1(\bmod p)$ in his identification of the $\operatorname{Spin}^{c}$ structures of $S_{p / q}^{3}(K)$ with $\mathbb{Z} / p \mathbb{Z}$. 
Proposition 4.4 For a fake right-handed trefoil knot $K$, we have the following computation of correction terms:

$$
\begin{gathered}
d\left(S_{(18 k+9) /(3 k+1)}^{3}(K), \mathfrak{s}_{0}\right)= \begin{cases}0, & \text { if } k \text { is even, } \\
+\frac{1}{2}, & \text { if } k \text { is odd. }\end{cases} \\
d\left(S_{(18 k+9) /(3 k+2)}^{3}(K), \mathfrak{s}_{0}\right)= \begin{cases}0, & \text { if } k \text { is even, } \\
-\frac{1}{2}, & \text { if } k \text { is odd. }\end{cases} \\
d\left(S_{(18 k+9) /(15 k+7)}^{3}(K), \mathfrak{s}_{0}\right)= \begin{cases}-2, & \text { if } k \text { is even, } \\
-\frac{3}{2}, & \text { if } k \text { is odd. }\end{cases} \\
d\left(S_{(18 k+9) /(15 k+8)}^{3}(K), \mathfrak{s}_{0}\right)= \begin{cases}-2, & \text { if } k \text { is even, } \\
-\frac{5}{2}, & \text { if } k \text { is odd. }\end{cases}
\end{gathered}
$$

Here $\mathfrak{s}_{0}$ is the unique spin $\operatorname{Spin}^{c}$ structure of the surgered three-manifold. And further, except for possibly $S_{(18 k+9) /(3 k+1)}^{3}(K) \cong-S_{(18 k+9) /(3 k+2)}^{3}(K)$, there are no other homeomorphisms between these manifolds.

Proof To compute the correction terms, we first compute that of the corresponding surgered manifolds on the unknot $U$. We use notation as in Section 2. For a linearly plumbed four-manifold, let $\left[S_{i}\right]$ be the homology class of the $i$-th exceptional sphere and $\phi_{i}=P D\left(\left[S_{i}\right]\right)$. Since $p=18 k+9$ is odd, there is a unique spin $\operatorname{Spin}^{c}$ structure, which, by abuse of notation, we always denote by $\mathfrak{s}_{0}$.

The lens space $S_{-9}^{3}(U)$ is the boundary of the linearly plumbed four-manifold $X=$ $(-9)$. The $\operatorname{Spin}^{c}$ structure $\mathfrak{t}_{0}$ on $X$ with $c_{1}\left(\mathfrak{t}_{0}\right)=\phi_{0}$ restricts to the spin $\operatorname{Spin}^{c}$ structure $\mathfrak{s}_{0}$ on $Y$. Hence $\operatorname{Char}\left(X, \mathfrak{s}_{0}\right)$ contains all cohomology classes of the form $(2 n+1) \phi_{0}$. We have

$$
\left((2 n+1) \phi_{0}\right)^{2}=(2 n+1)^{2} \phi_{0}^{2}=-9(2 n+1)^{2} \leq-9,
$$

hence $\quad d\left(S_{-9}^{3}(U), \mathfrak{s}_{0}\right)=\max _{K \in \operatorname{Char}\left(X, \mathfrak{s}_{0}\right)} \frac{K^{2}+\mathrm{rk} H^{2}(X)}{4}=\frac{(-9)+1}{4}=-2$.

The lens space $S_{-(18 k+9) /(3 k+1)}^{3}(U)(k \geq 1)$ can be thought of the boundary of the linearly plumbed four-manifold

$$
X=(-7,-2, \cdots,-2,-4) \text {, }
$$


where the number of -2 spheres is $k-1$. If $k$ is even, $\phi_{0}+\phi_{2}+\cdots+\phi_{k}$ is characteristic and

$\operatorname{Char}\left(X, \mathfrak{s}_{0}\right)=\left\{\sum_{i=0}^{k} C_{i} \phi_{i} \mid C_{0}, C_{2}, \cdots C_{k}\right.$ are odd while $C_{1}, C_{3}, \cdots C_{k-1}$ are even. $\}$

For $K=C_{0} \phi_{0}+\cdots+C_{k} \phi_{k} \in \operatorname{Char}\left(X, \mathfrak{s}_{0}\right)$, we have

$$
\begin{aligned}
K^{2} & =C_{0}^{2} \phi_{0}^{2}+2 C_{0} C_{1} \phi_{0} \phi_{1}+C_{1}^{2} \phi_{1}^{2}+2 C_{1} C_{2} \phi_{1} \phi_{2}+\cdots+2 C_{k-1} C_{k} \phi_{k-1} \phi_{k}+C_{k}^{2} \phi_{k}^{2} \\
& =-7 C_{0}^{2}+2 C_{0} C_{1}-2 C_{1}^{2}+2 C_{1} C_{2}-2 C_{2}^{2}+\cdots+2 C_{k-1} C_{k}-4 C_{k}^{2} \\
& =-6 C_{0}^{2}-\left(C_{0}-C_{1}\right)^{2}-\left(C_{1}-C_{2}\right)^{2}-\cdots-\left(C_{k-1}-C_{k}\right)^{2}-3 C_{k}^{2} .
\end{aligned}
$$

Now $C_{0}, C_{k}$ and all $C_{i}-C_{i+1}$ are odd numbers, so we get

$$
K^{2} \leq-6-1-\cdots-1-3=-9-k,
$$

and the equality can be obtained by setting the constants $C_{0}=C_{2}=\cdots=C_{k}=1$ and $C_{1}=C_{3}=\cdots=C_{k-1}=0$. Hence for $k$ even, we get

$d\left(S_{-(18 k+9) /(3 k+1)}^{3}(U), \mathfrak{s}_{0}\right)=\max _{K \in \operatorname{Char}\left(X, \mathfrak{s}_{0}\right)} \frac{K^{2}+\mathrm{rk} H^{2}(X)}{4}=\frac{(-9-k)+(k+1)}{4}=-2$.

If $k$ is odd, then $\phi_{1}+\phi_{3}+\cdots+\phi_{k}$ is characteristic and

$\operatorname{Char}\left(X, \mathfrak{s}_{0}\right)=\left\{\sum_{i=0}^{k} C_{i} \phi_{i} \mid C_{0}, C_{2}, \cdots C_{k-1}\right.$ are even while $C_{1}, C_{3}, \cdots C_{k}$ are odd. $\}$

As in the case where $k$ is even, for $K=C_{0} \phi_{0}+\cdots+C_{k} \phi_{k} \in \operatorname{Char}\left(X, \mathfrak{s}_{0}\right)$, we get

$$
\begin{aligned}
K^{2} & =-6 C_{0}^{2}-\left(C_{0}-C_{1}\right)^{2}-\left(C_{1}-C_{2}\right)^{2}-\cdots-\left(C_{k-1}-C_{k}\right)^{2}-3 C_{k}^{2} \\
& \leq-1 \cdots-1-3=-k-3 .
\end{aligned}
$$

Note that here we have $C_{0}$ is even and $C_{k}, C_{i}-C_{i+1}$ are odd, and the equality is obtained by letting $C_{0}=C_{2}=\cdots C_{k-1}=0$ and $C_{1}=C_{3}=\cdots=C_{k}=1$. And the correction term

$d\left(S_{-(18 k+9) /(3 k+1)}^{3}(U), \mathfrak{s}_{0}\right)=\max _{K \in \operatorname{Char}\left(X, \mathfrak{s}_{0}\right)} \frac{K^{2}+\mathrm{rk} H^{2}(X)}{4}=\frac{(-3-k)+(k+1)}{4}=-\frac{1}{2}$.

Altogether, we have

$$
d\left(S_{-(18 k+9) /(3 k+1)}^{3}(U), \mathfrak{s}_{0}\right)= \begin{cases}-2, & \text { if } k \text { is even, } \\ -\frac{1}{2}, & \text { if } k \text { is odd }\end{cases}
$$

Algebraic 83 Geometric Topology, Volume 6 (2006) 
By the properties of the corrections terms (1), we get

$$
d\left(S_{(18 k+9) /(3 k+1)}^{3}(U), \mathfrak{s}_{0}\right)= \begin{cases}2, & \text { if } k \text { is even, } \\ \frac{1}{2}, & \text { if } k \text { is odd. }\end{cases}
$$

The correction term $d\left(S_{(18 k+9) /(3 k+2)}^{3}(U), \mathfrak{s}_{0}\right)$ could be computed by thinking of the lens space $S_{-(18 k+9) /(3 k+2)}^{3}(U)$ as the boundary of $X=(-5,-2)$ if $k=0$ and of $X=(-6,-k-1,-3)$ if $k>0$. We get

$$
d\left(S_{(18 k+9) /(3 k+2)}^{3}(U), \mathfrak{s}_{0}\right)= \begin{cases}0, & \text { if } k \text { is even }, \\ \frac{3}{2}, & \text { if } k \text { is odd. }\end{cases}
$$

The correction term $d\left(S_{(18 k+9) /(15 k+7)}^{3}(U), \mathfrak{s}_{0}\right)$ is computed by thinking of the lens space $S_{-(18 k+9) /(15 k+7)}^{3}(U)$ as the boundary of

$$
X= \begin{cases}(-2,-2,-2,-3), & \text { if } k=0, \\ (-2,-2,-2,-2,-4,-2), & \text { if } k=1, \\ (-2,-2,-2,-2,-3,-3,-2), & \text { if } k=2, \\ (-2,-2,-2,-2,-3,-2, \cdots,-2,-3,-2), & \text { if } k \geq 3,\end{cases}
$$

where when $k \geq 3$, the number of $(-2)$ 's between each -3 is $k-2$. We get

$$
d\left(S_{(18 k+9) /(15 K+7)}^{3}(U), \mathfrak{s}_{0}\right)= \begin{cases}0, & \text { if } k \text { is even } \\ -\frac{3}{2}, & \text { if } k \text { is odd }\end{cases}
$$

The correction term $d\left(S_{(18 k+9) /(15 k+8)}^{3}(U), \mathfrak{s}_{0}\right)$ is computed by viewing the lens space $S_{-(18 k+9) /(15 k+8)}^{3}(U)$ as the boundary of

$$
X=(-2,-2,-2,-2,-2,-(k+2),-2,-2) \text {. }
$$

We have

$$
d\left(S_{(18 k+9) /(15 K+8)}^{3}(U), \mathfrak{s}_{0}\right)= \begin{cases}-2, & \text { if } k \text { is even, } \\ -\frac{1}{2}, & \text { if } k \text { is odd. }\end{cases}
$$

Now the correction term for $S_{p / q}^{3}(K)$ follows from Proposition 4.3.

By the properties of the correction terms (1), the only possible homeomorphisms are

$$
\begin{aligned}
S_{(18 k+9) /(3 k+1)}^{3}(K) & \cong-S_{(18 k+9) /(3 k+2)}^{3}(K), & & \text { for } k \geq 0, \\
S_{(18 k+9) /(3 k+1)}^{3}(K) & \cong S_{(18 k+9) /(3 k+2)}^{3}(K), & & \text { for } k \text { even, } \\
S_{(18 k+9) /(15 k+7)}^{3}(K) & \cong S_{(15 k+8) /(15 k+8)}^{3}(K), & & \text { for } k \text { even. }
\end{aligned}
$$

The last two possibilities can easily be ruled out by their Casson-Walker invariants. 
Our proof of Theorem 1.3 will use the Reidemeister torsion of a three-manifold. Refer to Milnor [19] or Turaev [33] for introductions on torsions. Turaev [32] and independently Sakai [31] computed the Reidemeister torsion of $S_{p / q}^{3}(K)$. The corresponding Reidemeister torsion of $S_{p / q}^{3}(K)$ for any $p$-th root of unity $\xi \neq 1$ is given by

$$
\tau\left(S_{p / q}^{3}(K), \xi\right)=\Delta_{K}(\xi) \cdot(\xi-1)^{-1}\left(\xi^{a}-1\right)^{-1},
$$

where $a$ is defined by $q a \equiv 1(\bmod p)$ and $\Delta_{K}(t)$ is the Alexander polynomial of $K$. In fact, this result is true for a knot in any homology sphere.

Proof of Theorem 1.3 Let $K$ be a genus one knot. Suppose $S_{p / q}^{3}(K) \cong \pm S_{p / q^{\prime}}^{3}(K)$, where $q \neq q^{\prime}$. By Theorem 1.2, we have

$$
\Delta_{K}(t)=t-1+t^{-1} .
$$

By a theorem of Turaev [33, Theorem 9.1], for any $p$-th root of unity $\xi \neq 1$, we have (for some $d \in \mathbb{Z} / p \mathbb{Z}$ )

$$
\tau\left(S_{p / q}^{3}(K), \xi\right)=\tau\left(S_{p / q^{\prime}}^{3}(K), \xi^{d}\right) .
$$

Here the equality is up to multiplication of $p$-th root of unity and sign. We have $(d, p)=1$, and by (18), we then get

$$
\left|\frac{\left(\xi-1+\xi^{-1}\right)}{(\xi-1)\left(\xi^{a}-1\right)}\right|=\left|\frac{\left(\xi^{d}-1+\xi^{-d}\right)}{\left(\xi^{d}-1\right)\left(\xi^{a^{\prime} d}-1\right)}\right|
$$

for any $p$-th root of unity $\zeta \neq 1$, where $a, a^{\prime}$ are defined by $q a \equiv 1, q^{\prime} a^{\prime} \equiv 1(\bmod p)$.

By Theorem 3.1, we have $a \equiv \pm a^{\prime}(\bmod p)$,

$$
\begin{aligned}
& p=12 \text { and } \quad\left\{ \pm a, \pm a^{\prime}\right\}=\{ \pm 1, \pm 5\} \quad(\bmod 12), \\
& \text { or } \quad p=18 k+9 \quad \text { and } \quad\left\{ \pm a, \pm a^{\prime}\right\}=\{ \pm(6 k+1), \pm(6 k+5)\} \quad(\bmod p)
\end{aligned}
$$

for some nonnegative integer $k$. Equivalently, we have $q \equiv \pm q^{\prime}(\bmod p)$,

$$
\begin{aligned}
& p=12 \text { and } \quad\left\{ \pm q, \pm q^{\prime}\right\}=\{ \pm 1, \pm 5\} \quad(\bmod 12), \\
& \text { or } \quad p=18 k+9 \quad \text { and } \quad\left\{ \pm q, \pm q^{\prime}\right\}=\{ \pm(3 k+1), \pm(3 k+2)\} \quad(\bmod p) .
\end{aligned}
$$

The $p=12$ case can be ruled out by direct computation of Casson-Walker invariants and Proposition 4.2.

By Theorem 1.2, Proposition 4.1 and Proposition 4.2, for $p=18 k+9$, we only need to consider the surgeries on fake right-handed trefoil knots with $q$ taking the four values: $3 k+1,3 k+2,15 k+7$ and $15 k+8$, and the surgeries on fake left-handed 
trefoil knots with $q$ taking the four values: $-(3 k+1),-(3 k+2),-(15 k+7)$ and $-(15 k+8)$.

Note that the $(p / q)$-surgery on a fake left-handed trefoil knot $K$ could be thought of as the $(-p / q)$-surgery on the mirror of $K$, which is a fake right-handed trefoil knot. Now the theorem follows from Proposition 4.4.

Remark Note that in the genus one case, we rule out cosmetic surgeries of slopes with opposite signs by Theorem 1.2. However, for the genus two knot $K=9_{44}, S_{+1}^{3}(K)$ and $S_{-1}^{3}(K)$ have the same Heegaard Floer homology, though the two surgered manifolds are distinguished by their hyperbolic volumes [23]. Thus, Theorem 1.2 can not be established for genus two knots (just using Heegaard Floer theory). So for genus two knots, our method might be applied to rule out truly cosmetic surgeries of slopes with the same sign, but there still might be truly cosmetic surgeries of slopes with opposite signs.

For knots in higher Seifert genus, our method might not work (even to rule out truly cosmetic surgeries with same sign slopes). Our method is to write the Reidemeister torsions of surgered manifolds on a knot admitting $L$-space surgeries as products of cyclotomic numbers and use number theoretic results to get restrictions on possible cosmetic surgery slopes (in favor of Theorem 1.2). As is pointed out in Kadokami [15], the $(-2,3,7)$-pretzel knot $K$ has Alexander polynomial

$$
\Delta_{K}(t)=t^{10}-t^{9}+t^{7}-t^{6}+t^{5}-t^{4}+t^{3}-t+1,
$$

which is not a product of cyclotomic polynomials. And Fintushel and Stern [8] showed that 18 and 19 surgeries along $K$ yield lens spaces. So we might need more machinery.

\section{References}

[1] Problems in low-dimensional topology, from: "Geometric topology (Athens, GA, 1993)”, (R Kirby, editor), AMS/IP Stud. Adv. Math. 2, Amer. Math. Soc., Providence, RI (1997) 35-473 MR1470751

[2] T M Apostol, Introduction to analytic number theory, Springer, New York (1976) MR0434929

[3] S A Bleiler, C D Hodgson, J R Weeks, Cosmetic surgery on knots, from: "Proceedings of the Kirbyfest (Berkeley, CA, 1998)", Geom. Topol. Monogr. 2, Geom. Topol. Publ., Coventry (1999) 23-34 MR1734400

[4] G de Rham, S Maumary, MA Kervaire, Torsion et type simple d'homotopie, Exposés faits au séminaire de Topologie de l'Université de Lausanne. Lecture Notes in Mathematics, No. 48, Springer, Berlin (1967) MR0222893 
[5] R Dvornicich, On an equation in cyclotomic numbers, Acta Arith. 98 (2001) 71-94 MR1831457

[6] E Eftekhary, Longitude Floer homology and the Whitehead double, Algebr. Geom. Topol. 5 (2005) 1389-1418 MR2171814

[7] V Ennola, On relations between cyclotomic units, J. Number Theory 4 (1972) 236-247 MR0299585

[8] R Fintushel, R J Stern, Constructing lens spaces by surgery on knots, Math. Z. 175 (1980) 33-51 MR595630

[9] W Franz, Uber die Torsion einer Überdeckung, J. Reine Angew. Math. 173 (1935) 245-254

[10] D Gabai, Foliations and the topology of 3-manifolds. II, J. Differential Geom. 26 (1987) 461-478 MR910017

[11] P Ghiggini, Knot Floer homology detects genus-one fibred links arXiv: math.GT/0603445

[12] C M Gordon, J Luecke, Knots are determined by their complements, J. Amer. Math. Soc. 2 (1989) 371-415 MR965210

[13] M Hedden, On knot Floer homology and cabling, Algebr. Geom. Topol. 5 (2005) 1197-1222 MR2171808

[14] M Jankins, W D Neumann, Lectures on Seifert manifolds, volume 2 of Brandeis Lecture Notes, Brandeis University, Waltham, MA (1983) MR741334

[15] T Kadokami, Reidemeister torsion of homology lens spaces, from: "Proceedings of the east asian school of knots, links, and related topics (Seoul, Korea)" (2004)

[16] P B Kronheimer, T Mrowka, PS Ozsváth, Z Szabó, Monopoles and lens space surgeries arXiv:math.GT/0310164

[17] M Lackenby, Dehn surgery on knots in 3-manifolds, J. Amer. Math. Soc. 10 (1997) 835-864 MR1443548

[18] Y Mathieu, Closed 3-manifolds unchanged by Dehn surgery, J. Knot Theory Ramifications 1 (1992) 279-296 MR1180402

[19] J Milnor, Whitehead torsion, Bull. Amer. Math. Soc. 72 (1966) 358-426 MR0196736

[20] Y Ni, Closed 3-braids are nearly fibred arXiv:math.GT/0510243

[21] Y Ni, Knot Floer homology detects fibred knots arXiv:math.GT/0607156

[22] Y Ni, Sutured Heegaard diagrams for knots, Algebr. Geom. Topol. 6 (2006) 513-537 MR2220687

[23] P Ozsváth, Z Szabó, Knot Floer homology and rational surgeries arXiv: math.GT/0504404

Algebraic 83 Geometric Topology, Volume 6 (2006) 
[24] P Ozsváth, Z Szabó, Absolutely graded Floer homologies and intersection forms for four-manifolds with boundary, Adv. Math. 173 (2003) 179-261 MR1957829

[25] P Ozsváth, Z Szabó, On the Floer homology of plumbed three-manifolds, Geom. Topol. 7 (2003) 185-224 MR1988284

[26] P Ozsváth, Z Szabó, Heegaard diagrams and holomorphic disks, from: "Different faces of geometry”, Int. Math. Ser. (N. Y.) 3, Kluwer/Plenum, New York (2004) 301-348 MR2102999

[27] P Ozsváth, Z Szabó, Holomorphic disks and topological invariants for closed threemanifolds, Ann. of Math. (2) 159 (2004) 1027-1158 MR2113019

[28] P Ozsváth, Z Szabó, Knot Floer homology, genus bounds, and mutation, Topology Appl. 141 (2004) 59-85 MR2058681

[29] Y W Rong, Some knots not determined by their complements, from: "Quantum topology”, Ser. Knots Everything 3, World Sci. Publ., River Edge, NJ (1993) 339-353 MR1273583

[30] R Rustamov, The renormalized Euler characteristic and L-space surgeries arXiv: math.GT/0506320

[31] T Sakai, Reidemeister torsion of a homology lens space, Kobe J. Math. 1 (1984) 47-50 MR784347

[32] V G Turaev, Reidemeister torsion and the Alexander polynomial, Mat. Sb. (N.S.) 18(66) (1976) 252-270 MR0433462

[33] V Turaev, Introduction to combinatorial torsions, Lectures in Mathematics ETH Zürich, Birkhäuser Verlag, Basel (2001) MR1809561

[34] L C Washington, Introduction to cyclotomic fields, second edition, Graduate Texts in Mathematics 83, Springer, New York (1997) MR1421575

Department of Mathematics, University of California at Berkeley

Berkeley, CA 94720, USA

wang@math . berkeley.edu

Received: 26 February 2006 Revised: 26 July 2006 